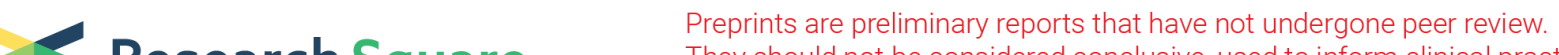 Research Square They should not be considered conclusive, used to inform clinical practice, or referenced by the media as validated information.
}

\section{Ultrasound image patterns right after birth can predict healthy neonates - a nested case-control study}

\section{Guannan Xi}

Obstetrics and Gynecology Hospital of Fudan University https://orcid.org/0000-0002-6888-5613 Jiale Dai

Obstetrics and Gynecology Hospital of Fudan University

\section{Xuefeng Wang}

Obstetrics and Gynecology Hospital of Fudan University

\section{Fei Luo}

Obstetrics and Gynecology Hospital of Fudan University

\section{Chenggqiu Lu}

Obstetrics and Gynecology Hospital of Fudan University

\section{Yun Yang}

Obstetrics and Gynecology Hospital of Fudan University

Jimei Wang ( $\nabla$ wjm8219@163.com )

Obstetrics and Gynecology Hospital of Fudan University

\section{Research article}

Keywords: Lung ultrasound; Neonatal adaptation; Pulmonology; Respiratory function

Posted Date: July 16th, 2020

DOl: https://doi.org/10.21203/rs.3.rs-42000/v1

License: (c) (1) This work is licensed under a Creative Commons Attribution 4.0 International License. Read Full License 


\section{Abstract}

Background Lungultarsound(LUS) is widely used to diagnose neonatal lung diseases, yet image patterns on intrauterine to extrauterine stage(right after birth), of which impairment is well related to lung disease, remains unclear.

Objectives To identify these image patterns that can distinguish healthy infants from infants with lung disease.

Methods This is a nested case-control study in a top-ranking obstetrics hospital in China, between 1 January 2020 to 1 April 2020. Infants transferred to the NICU after birth who had LUS obtained at 0.5, 1, 2, 4, 6 hours time intervals were enrolled. Confirmed by 3-day follow-up, case and control groups contains 22 patients and 473 healthy infants. Their GA ranges from 33.5 to 41.0 weeks. A newly designed protocol was used to capture the LUS image. The image patterns and their variations were shown and categorized as high and low-risk groups. The predictive value for healthy infants and patients were calculated.

Rsults Low-risk patterns, accompanied with no high-risk ones, typically appeared in healthy infants (specificity $=86.4 \%, \mathrm{PPV}=99.0 \%$ ), whereas four high-risk patterns could be seen in both healthy infants and patients (specificity $=62.4 \%, \mathrm{PPV}=9.6 \%$ ). High-risk patterns were more likely to be pathological signs when appearing at the oxter and lower back and to be physiological signs when appearing at the prothorax.

Conclusions LUS is valid to differentiate healthy infants from potential patients shortly after birth. Infants with low-risk patterns only are highly likely to be healthy, whereas infants with high-risk patterns have a risk for respiratory issues but need prolonged monitoring to confirm.

\section{Introduction}

Neonates' lung transition stage(from intrauterine to extrauterine), the process of fluid clearance and alveolar inflation at the early stage after birth (defined as 6 hours in our research), is complicated, and its impairment has been related to pulmonary diseases such as neonate respiratory distress (RDS) and transient tachypnea of newborn (TTN)[1-3]. However, it is sometimes difficult to distinguish healthy infants from those with lung diseases at this stage, since they can both present nonspecific symptoms such as short apnea, mild anhelation, and transient cyanosis.

Lung ultrasound (LUS) is widely used in neonate intensive care units (NICUs) worldwide. It is a valid modality for the diagnosis of some neonate lung diseases, for example, RDS, TTN, and meconium aspiration syndrome(MAS)[4-6]. Recently, many studies have begun to focus on predictive usefulness in respiratory care. These researches assessed the predictive value of LUS score, and find it useful to predict the need for surfactant[7], intubation[8], and ventilation[9] in neonates of variable GA. Nevertheless, combined the published reports and our experience, when an infant has a low score(such as $3 \sim 4$ scores only have a specificity of $25 \%[10])$, current score system seems to be not enough effective to make a 
practical decision, especially in the neonates shortly after birth. This dilemma may cause by the lung fluid clearance delay mentioned above, which may lead to confusion between actual physiological LUS images and pathological ones(retrospective confirmed). For example, in our pilot study, there were some infants with pathological signs(such as "consolidation" and "dense B-line", which was regarded as signs of MAS[11] and TTN[12])were verified to be healthy later.

To make a quick and definite decision that whether a neonate with mild respiratory symptoms needs further medical care at the early stage after birth, it's essential to address the confusion mentioned above. So we conducted this nested case-control study to describe these patterns (on the ground of our pilot study, we grouped these patterns into high-risk and low-risk patterns, definition seen in the method) and assessed the predictive value of them.

\section{Materials And Methods}

Study objectives and design This is a nested case-control study that comprised 495 neonates(473 infants in control group and 22 infants with lung diseases in case group, confirmed retrospectively) in the NICU of the Obstetrics \& Gynecology Hospital of Fudan University, Shanghai, China, from 1 January 2020 to 1 April 2020.

All infants delivered in the obstetric department were routinely transferred to NICU observation ward for termporarily monitoring(no more than 6 hours) in case of potential diseases. During the study period, these infants enrolled consequtively no matter they with or without respiratory symptoms and some of them were excluded as following criteria: खabsence of complete and qualified clinic data or ultrasound images; \with cardiac issues that is diagnosed after admission to NICU. As our pilot studies showed that some infants with previously considered pathological LUS image patterns(mentioned in the introduction) were confirmed to be healthy, we made every infant enrolled in this study received LUS inspection to acquire all possible kinds of patterns in healthy infants. The images were collected with a newly designed scanning protocol(seen as following) at a predetermined time ( 0.5 hours, 1 hour, 2 hours, 4 hours, or 6 hours after birth,).

Because the diagnosis of most respiratory diseases of neonates are based on CXR that generally might be done only when infants have severe respiratory difficulty, determining healthy infants shortly after birth is difficult. So we adopted the nested case-control design that collecting data of all participants right first and decided the case and control groups after all patients were diagnosed.

Scanning protocol Lung ultrasound was routinely performed at bedside using a Sparq Ultrasound System (Philips Healthcare, Andover, MA) equipped with a 3-13 MHz linear array transducer and concurrently reported using a reporting template within the ICU electronic patient record. To acquire a constant (between different inspectors and different inspections) and comprehensive description of the neonates' lungs, a new scanning protocol was designed and applied. We improved the conventional scanning protocol[13] in which the probe scans continually over 6 lung regions to a new protocol in which the probe scans at 20 predetermined points (shown in Fig. S1). 
Defining RDS, TTN, congenital pneumonia, pneumothorax and healthy infants

RDS was defined in two ways: using a combination of chest radiography (Berlin-CXR)[14] and the PS application threshold recommended by the European Consensus Guidelines[15].

TTN is a clinical diagnosis and is supported by findings from chest radiographs, such as increased lung volumes with flat diaphragms and mild cardiomegaly [16].

Congenital pneumonia was diagnosed based on comprehensive evidence[17] from complete blood counts, C-reactive proteins, cultures for main types of pathogens (listed in the reference), as well as findings on CXR.

PTX was mainly confirmed by clinical features, LUS, and closed thoracic drainage. LUS, which is believed to have higher sensitivity than CRX[18, 19].

These diagnosis are made by the experienced neonatology specilist in this study team. To acquire the Xray evidence mentioned above, suspected patients were routinely inspected by a technician, and the conclusions were drawn by a junior doctor and verified by a senior doctor from the radiology department.

\section{Healthy infants}

After excluding the diseases mentioned above, infants were regarded as healthy and confirmed on 3-day follow-up. However, regarding mild TTN that can be a physiologic diagnosis needs no further medical care and hard to differentiate, we classified these infants into control group so that the conclusion of this study is pratical.

\section{Low-risk and high-risk image patterns}

Previous studies have indicated that "A-line"[4], "small amounts, and a large amount of B-line"[20](defined as "coalescence B line" in this reference) is normal patterns for neonates, and has regarded "compact Bline", "dense B-line"(or defined as "white lung" in these references), "consolidation" as abnormal patterns[4, $2,21,12,22]$. However, in our pilot study, either supposed normal or abnormal patterns can be seen both in healthy infants and patients. To make our conclusion, to which physicians can make a definite and timely decision according, practical, we regarded the patterns as high-risk and low-risk instead of simply naming as "normal" or "abnormal".

To clarify different B-line patterns(shown in Fig. 3) and its various significance for lung diseases, especially the "large amount of DB"(low risk) and "compact B-line"(high risk), "dense B-line"(high risk), we characterized the low-risk patterns of B-lines(distributed B-lines) as"can be discriminated against each other". This can be very useful when assessing neonates on dynamic LUS according to our experience.

\section{Statistical analysis}

Data was shown as frequencies or percentages and as the means and standard deviations or medians and interquartile ranges according to distribution. Differences between the groups were compared by the 
chi-squared or Fisher's exact test for categorical variables and Student's t-test or Mann-Whitney U test for continuous variables, depending on the distribution. Sensitivity, specificity, LR, PPV and NPV were calculated to evaluate the predictive value of LUS patterns. A nominal 2-sided probability value $<0.05$ was considered to indicate statistical significance. All of the calculations were performed using SPSS 23.0 (SPSS Inc. Chicago, IL).

\section{Result}

\section{Participants and LUS images}

During the 4-month study period, out of 504 NICU admissions, 495 infants were analyzed, 9 infants were excluded for absence of data (shown in Fig. 1). The case group has 4 patients with RDS; 7 infants with congenital pneumonia ( 3 infected by Escherichia coli; 2 infected by mycoplasma; 2 were not pathogenpositive but recovered after application of antibiotics); and 8 infants with TTN or mild RDS (since they are difficult to differentiate) as well as PTX infants (confirmed by CXR and closed thoracic drainage). The control group consists of 473 infants confirmed to be healthy retrospectively. Regarding baseline characteristics, healthy infants contained more males $(246,52.0 \%$ vs $6,27.3 \%, p=0.02)$, whereas the patient group had a higher proportion of preterm births $(29,6.1 \% \mathrm{vs} 9,45.5 \%, p<0.00)$. In addition, the patient group had relatively more LBW infants $(11,13.6 \%$ vs $3,2.3 \%, p=0.02)$. There was no significant difference between the two groups in terms of maternal age, gestational age, rate of meconium-stained amniotic fluid (MSAF), or premature rupture of fetal membrane (PROM).

\section{Four low-risk image patterns and four high-risk patterns}

Eight image patterns were found in all infants(shown in Fig. 2, Table 2a), which can be categorized as high-risk patterns and low-risk patterns(shown in Fig. 2). 
Table 1

Baseline charicaterics of healthy infants and patients

\begin{tabular}{lll} 
Characteristic & $\begin{array}{l}\text { Case group } \\
(\mathbf{4 7 3} \text { healthy infants) }\end{array}$ & $\begin{array}{l}\text { Control group } \\
(\mathbf{2 2} \text { patients) }\end{array}$ \\
\hline Diagnosis for admission & Nomal & RDS (4/22); \\
& congenital pneumonia $(7 / 22) ;$ \\
& TTN or mild RDS(8/22) \\
& PTX (3/101)
\end{tabular}

\section{Maternal characteristics}

Maternal age

$39 \pm 9$

$38 \pm 12$

(median \pm a quater)

Advanced maternal age $(n, \%)^{\dagger}$

$343(72.5 \%)$

$14(63.6 \%)$

$\mathrm{GA}($ mean $\pm \mathrm{SD})$

$39.2 \pm 1.4$

$38.1 \pm 2.2$

Delivery $(\mathrm{n}, \%)$

$271(57.3 \%)$

$13(59.1 \%)$

vaginal birth

$191(40.4 \%)$

$8(36.4 \%)$

C-section

$11(2.3 \%)$

$1(4.5 \%)$

Forceps delivery

\section{Infants characteristics}

Gender(male) (n, \%)

$246(52.0 \%)$

$6(27.3 \%)$ *

Preterm birth $(\mathrm{n}, \%)$

$29(6.1 \%)$

$19(45.5 \%)$ *

Birth weight

$3338 \pm 411$

$3174 \pm 573$

$(g$, mean $\pm S D)$

$\operatorname{LBW}^{\ddagger}(\mathrm{n}, \%)$

$\operatorname{MSAF}(n, \%)$

No

$1^{\circ}$

$11^{\circ}$

$111^{\circ}$

PROM (n, \%)

PROM time to birth ${ }^{\dagger}$
$11(2.3 \%)$

383 (88.2\%)

$11(2.3 \%)$

$13(2.7 \%)$

$32(6.8 \%)$

$88(18.7 \%)$

$3(13.6 \%)$ 


\begin{tabular}{|c|c|c|}
\hline Characteristic & $\begin{array}{l}\text { Case group } \\
\text { (473 healthy infants) }\end{array}$ & $\begin{array}{l}\text { Control group } \\
\text { (22 patients) }\end{array}$ \\
\hline$<8$ hours $(\mathrm{n}, \%)$ & $22(4.7 \%)$ & $0(0.0 \%)$ \\
\hline$>8$ hours $(\%)$ & $66(14.0 \%)$ & $3(13.6 \%)$ \\
\hline \multicolumn{3}{|c|}{ SD: standard deviation; LBW: low birth weight; PTX: pneumothorax; } \\
\hline \multicolumn{3}{|c|}{ TTN: transient tachypnea of newborn; RDS: respiratory distress; } \\
\hline \multicolumn{3}{|c|}{ MSAF: meconium-stained amniotic fluid; } \\
\hline \multicolumn{3}{|c|}{ PROM: premature rupture of fetal membranes } \\
\hline \multicolumn{3}{|c|}{ Preterm birth was defined as gestation age is smaller than 37 weeks. } \\
\hline \multicolumn{3}{|c|}{ † Advanced maternal age: maternal age is greater than 35 years. } \\
\hline \multicolumn{3}{|c|}{ ¥ Low birth weight means the birth weight is lower than $2500 \mathrm{~g}$. } \\
\hline \multicolumn{3}{|c|}{$\begin{array}{l}\text { † As our routine, the PROM time greater than } 8 \text { hours is a risk factor for infection of neonates, which } \\
\text { means a higher risk of pneumonia. }\end{array}$} \\
\hline $\begin{array}{l}* \text { there is a signi } \\
\text { since they are ca }\end{array}$ & 5 ; Gender, Preterm bi & $3 W$ are performed chi-squared test \\
\hline
\end{tabular}


Table 2

a. LUS patterns in healthy ifants and lung diseases infants

\begin{tabular}{|c|c|c|c|c|c|c|c|}
\hline \multirow[t]{2}{*}{ LUS patterns } & \multirow[t]{2}{*}{ Healthy infants } & \multicolumn{6}{|c|}{ Lung diseases } \\
\hline & & RDS & \multicolumn{2}{|c|}{ TTN or mild RDS } & $\mathrm{CP}$ & PTX & Total \\
\hline Only & $295 / 473$ & $0 / 4$ & \multicolumn{2}{|l|}{$2 / 8$} & $0 / 7$ & $1 / 3$ & $3 / 22$ \\
\hline \multicolumn{8}{|l|}{ low risk } \\
\hline Only & $0 / 473$ & $0 / 4$ & $0 / 8$ & & $0 / 7$ & $0 / 3$ & $0 / 22$ \\
\hline \multicolumn{8}{|l|}{ high risk } \\
\hline Both & $178 / 473$ & $4 / 4$ & $6 / 8$ & & $7 / 7$ & $2 / 3$ & $19 / 22$ \\
\hline \multicolumn{8}{|c|}{ low and high risk } \\
\hline \multicolumn{8}{|c|}{ Table 2b. Reliability of "Only low risk" pattern to predict healthy infants and patients } \\
\hline \multirow[t]{2}{*}{ LUS patterns } & \multicolumn{2}{|l|}{$\%$} & \multicolumn{2}{|l|}{ LR } & \multirow[t]{2}{*}{ PPV } & & \multirow[t]{2}{*}{ NPV } \\
\hline & sensitivity & specificity & positive & negative & & & \\
\hline Only & 62.4 & 86.4 & 4.6 & 0.4 & $99.0 \%$ & & $9.6 \%$ \\
\hline \multicolumn{8}{|l|}{ low risk ${ }^{\dagger}$} \\
\hline Both & 86.4 & 62.4 & 2.3 & 0.2 & $9.6 \%$ & & $99.0 \%$ \\
\hline \multicolumn{8}{|c|}{ low and high risk ${ }^{\dagger \dagger}$} \\
\hline \multicolumn{8}{|c|}{ LUS: lung ultrasound; RDS: respiratory distress; TTN: transient tachypnea of newborn; } \\
\hline \multicolumn{8}{|c|}{ CP: congenital pneumonia; PTX: peumothorax; LR: likelihood ratio; } \\
\hline \multicolumn{8}{|c|}{ PPV: positive predictive value; NPV: negative predictive value } \\
\hline \multicolumn{8}{|c|}{$\begin{array}{l}\text { + When caculating sensitivtiy, specificity, LR, PPV and NPV for healthy infants, the "true positive" is } \\
\text { defined as healthy infants with only low risk LUS image pattens and "true negative" in defined as } \\
\text { patints with both low and high risk patterns(due to no patient is with "only high-risk pattern"). }\end{array}$} \\
\hline
\end{tabular}

The pure existence (without any high-risk patterns) of the four low-risk patterns could only be seen primarily in healthy infants (specificity $=86.4 \%, \mathrm{PPV}=99.0 \%$ ). However, the high-risk patterns could be seen in both healthy infants and patients (specificity $=62.4 \%$, PPV $=9.6 \%$; Table $2 \mathrm{~b}$ ).

In addition, when the high-risk patterns appeared in the lower back (positions 12, 14 and 18, and 20), they were highly likely signs of RDS or TTN (12/12, Table S1). In contrast, when these theree patterns were 
detected only in the lower or upper part of the prothorax, they were likely to be normal signs. Besides, for patients, some low-risk patterns also can be seen in some predetermined positions, such as upper of the prothorax, lower prothorax, oxter, and lower back( Table S1).

\section{LUS image patterns of transition in healthy infants at early stages after birth}

The proportion of the patterns were constant generally at different times $(0.5 \mathrm{~h}, 1 \mathrm{~h}, 2 \mathrm{~h}, 4 \mathrm{~h}$, and $6 \mathrm{~h}$ after birth) ( $p>0.05$ for each type of image between groups, Mann-Whitney $U$ test), except for the patterns at 6 hours (shown in Fig. 3). Although it appeared that the 1-hour and 2-hour patterns showed more instances of "small amount of DBs" than the 6-hour patterns, there was no significant difference $(p>0.05$, MannWhitney U test). However, "irregular consolidation with DBs" appeared more frequently at the 6th hour than at the 4 th hour $(p=0.045$, Mann-Whitney $U$ test). High-risk patterns and low-risk patterns were not significantly different except at 0.5 hours compared with 4 hours. ( $6 \mathrm{~h}$ vs $0.5 \mathrm{~h}, \mathrm{p}=0.51 ; 4 \mathrm{~h}$ vs $0.5 \mathrm{~h}, \mathrm{p}=$ 0.042, Mann-Whitney U test).

\section{Discussion}

\section{Findings and interpretation}

This study has two clinically relevant findings. (1) The typical pathological patterns reported previously ("large amount of B-lines", "compact B lines", "dense B-lines", "irregular consolidation with DBs", and "mild consolidation with air bronchograms") may also manifest in healthy neonates right after birth. This may be because the delay lung fluid clearance that might leave some alveoli remaining uninflated and full of fluid, from which the "compact B line" and "consolidation" signs originate[23-25]. (2) The pure existence of only the low-risk LUS patterns ("purely A-lines", small amount of B-lines", "moderate amount of B-lines", and "large amount of B-lines") can be regarded as strong evidence of healthy lungs. The high-risk patterns indicated potential lung diseases according to their position. Nevertheless, why the pattern positions have various predictive value remains unclear.

\section{Comparison with other studies}

The lung passes through three distinct phases as it transitions from a liquid-filled organ with low blood flow into the sole organ of oxygen exchange after birth[26]. Thus, LUS image patterns are dynamic and complex, different from those in adults or neonates at later days after birth. A study described the aeration and fluid clearance of neonate lungs during the first $10 \mathrm{~min}$ to 24 hours of life[20]. Their results showed that patterns of "coalescence of B-lines" (similar to pattern D, E or F), "sharp pleural line with Blines", and "sharp pleural line only A-lines" normally exist in the neonatal lung. What is different and new in our study is that consolidation ( $\mathrm{G}$ and $\mathrm{H}$ ) can also be found in infants confirmed to be healthy later. This may come from our new scanning protocol, which detects signals from the upper back (positions 11, 13 and 17, 19) where these "normal consolidation" patterns often exists. Combined with "consolidation" often being regarded as a sign of pneumonia[22], our findings suggest the need of a prolonged monitor to obtain harder evidence of pneumonia to prevent overdiagnosis. 
The full hyperechoic image of the lung fields or "white lung" (corresponding to pattern E or F in Fig. 2) indicated a failure of infants to adapt and can be a predictor of the need for respiratory support (sensitivity $77.7 \%$, specificity $100 \%$ )[27]. However, in our study, this pattern was also seen in a large proportion of healthy infants $(178 / 473$, Table 2$)$. This difference originated from our more detailed scanning protocol, which paid more attention to the upper back.

Brat and colleagues proposed a scoring system to forecast the need for respiratory treatment[10]. Their system has a high NPV but a low PPV (93 vs. 20). This could lead to the same conclusion as ours that LUS can predict healthy infants more effectively than lung diseases. However, what we think might need to be improved is to capture images at more and specific points to ensure consistency of diagnosis, as we did in this study.

Strengths and Limitations of our study: To our knowledge, this is the first study concerned with the relation between scanning positions and different LUS image patterns. To shed light on this problem, we improved the current scanning method to capture images at 20 predetermined points on the chest wall. With this detailed protocol, we found that "consolidation" may be a physiological sign at upper back positions. In addition, following the scanning protocol in order from position 1 to 20 (shown in fig. S1), an LUS examination can be accomplished in approximately only $6 \mathrm{~min}$ and for 3 positions (supine position, left lateral position, and right position). So the LUS can be a quick and safe screening method for every infant with any respiratory difficulty after birth. Another advantage is the nested case-control design we adopted: as some lung diseases are commonly diagnosed based on radiology evidence, it is difficult to confirm healthy infants shortly after birth. To solve this problem, we collected LUS images from all participants but did not analyze them until all patients were diagnosed.

Nevertheless, there are some limitations in our study. Most significant is the potential bias of specificity, sensitivity, etc. As we enrolled neonates born in our hospital (an advanced obstetrics and gynecology hospital in China) consecutively, the patients were only a small proportion of them, which may lead to insufficient patients for the case group. To address this problem, we are conducting further research enrolling in more patients.

\section{Conclusion}

LUS is valid to differentiate healthy infants from potential patients shortly after birth. Infants with low-risk patterns only are highly likely to be healthy, whereas infants with high-risk patterns have a risk for respiratory issues but need prolonged monitoring to confirm.

\section{List Of Abbreviations}

LUS: lung ultrasound; RDS: respiratory distress;

TTN: transient tachypnea of newborn; NICU: the neonatal intensive care unit; 
CP: congenital pneumonia; PTX: peumothorax;

CXR: chest X-ray;RR: respiratory rate

TcSO2: transcutaneous oxygen saturation; DB:distributed B-line

MAS: meconium aspiration syndrome; MSAF: meconium-stained amniotic fluid;

PROM: premature rupture of fetal membranes; SD: standard deviation

LR: likelihood ratio; PPV: positive predictive value;

NPV: negative predictive value

LBW:low birth weight;

\section{Declarations}

Ethics approval and consent to participate This study was approved by the ethics committee of Obstetrics and Gynecology Hospital of Fudan University (No. Kyy-2020-162). Informed consent was obtained from the parents of the babies for using the images and data for analysis.

Consent for publication Not applicable.

Competing interests There is no conflict of interest associated with this manuscript.

Funding This research was funded by financial support from the Shanghai Municipal Health commission, China.

Author Contributions M.D JMW and Doctor GNX proposed the idea of this research and designed the protocol. Doctor GNX and JLD, performed the data acquisition and analyses. Doctor GNX and M.D JLD drafted the article and revising it critically for important intellectual content. FL, YY, CQL, XFW have been involved in revising the manuscript critically for important intellectual content. All authors read and approved the final manuscript.

Acknowledgement Baoyunlei and Yinjun,the clerks of our department, who cannot be included in the authorship must be appreciated because of great efforts to this paper.

\section{References}

1. Roth-Kleiner M, Wagner BP, Bachmann D, Pfenninger J. Respiratory distress syndrome in near-term babies after caesarean section. SWISS MED WKLY. 2003;133(19-20):283-8. 'doi:'2003/19/smw10121. 
2. Liu J, Chen XX, Li XW, Chen SW, Wang Y, Fu W. Lung Ultrasonography to Diagnose Transient Tachypnea of the Newborn. CHEST. 2016;149(5):1269-75. 'doi:'10.1016/j.chest.2015.12.024.

3. Helve O, Pitkanen O, Janer C, Andersson S. Pulmonary fluid balance in the human newborn infant. NEONATOLOGY. 2009;95(4):347-52. 'doi:'10.1159/000209300.

4. Corsini I, Parri N, Ficial B, Dani C. Lung ultrasound in the neonatal intensive care unit: Review of the literature and future perspectives,2020.

5. Kurepa D, Zaghloul N, Watkins L, Liu J. Neonatal lung ultrasound exam guidelines. J PERINATOL. 2018;38(1):11-22. 'doi:'10.1038/jp.2017.140.

6. Mazmanyan P, Kerobyan V, Shankar-Aguilera S, Yousef N, De Luca D. Introduction of point-of-care neonatal lung ultrasound in a developing country. EUR J PEDIATR. 2020. 'doi:'10.1007/s00431-02003603-w.

7. De Martino L, Yousef N, Ben-Ammar R, Raimondi F, Shankar-Aguilera S De Luca D. Lung Ultrasound Score Predicts Surfactant Need in Extremely Preterm Neonates. PEDIATRICS. 2018;142(3). 'doi:'10.1542/peds.2018-0463.

8. Raimondi F, Migliaro F, Sodano A, Ferrara T, Lama S, Vallone G, Capasso L. Use of neonatal chest ultrasound to predict noninvasive ventilation failure. PEDIATRICS. 2014;134(4):e1089-94. 'doi:'10.1542/peds.2013-3924.

9. Rodriguez-Fanjul J, Balcells C, Aldecoa-Bilbao V, Moreno J, Iriondo M. Lung Ultrasound as a Predictor of Mechanical Ventilation in Neonates Older than 32 Weeks. NEONATOLOGY. 2016;110(3):198-203. 'doi:'10.1159/000445932.

10. Brat R, Yousef N, Klifa R, Reynaud S, Shankar AS De Luca D. Lung Ultrasonography Score to Evaluate Oxygenation and Surfactant Need in Neonates Treated With Continuous Positive Airway Pressure. JAMA PEDIATR. 2015;169(8):e151797. 'doi:'10.1001/jamapediatrics.2015.1797.

11. Piastra M, Yousef N, Brat R, Manzoni P, Mokhtari M, De Luca D. Lung ultrasound findings in meconium aspiration syndrome. EARLY HUM DEV. 2014;90 Suppl 2:S41-3. 'doi:'10.1016/S03783782(14)50011-4.

12. Raimondi F, Yousef N, Rodriguez FJ, De Luca D, Corsini I, Shankar-Aguilera S, Dani C, Di Guardo V, Lama S, Mosca F, Migliaro F, Sodano A, Vallone G, Capasso L. A Multicenter Lung Ultrasound Study on Transient Tachypnea of the Neonate. NEONATOLOGY. 2019;115(3):263-8. 'doi:'10.1159/000495911.

13. Liu J, Copetti R, Sorantin E, Lovrenski J, Rodriguez-Fanjul J, Kurepa D, Feng X, Cattaross L, Zhang H, Hwang M, Yeh TF, Lipener Y, Lodha A, Wang JQ, Cao HY, Hu CB, Lyu GR, Qiu XR, Jia LQ, Wang XM, Ren XL, Guo JY, Gao YQ, Li JJ, Liu Y, Fu W, Wang Y, Lu ZL, Wang HW, Shang LL. Protocol and Guidelines for Point-of-Care Lung Ultrasound in Diagnosing Neonatal Pulmonary Diseases Based on International Expert Consensus. J Vis Exp. 2019(145). 'doi:'10.3791/58990.

14. Ranieri VM, Rubenfeld GD, Thompson BT, Ferguson ND, Caldwell E, Fan E, Camporota L, Slutsky AS. Acute respiratory distress syndrome: the Berlin Definition. JAMA. 2012;307(23):2526-33. 'doi:'10.1001/jama.2012.5669. 
15. Sweet DG, Carnielli V, Greisen G, Hallman M, Ozek E, Te PA, Plavka R, Roehr CC, Saugstad OD, Simeoni U, Speer CP, Vento M, Visser G, Halliday HL. European Consensus Guidelines on the Management of Respiratory Distress Syndrome - 2019 Update. NEONATOLOGY. 2019;115(4):432 50. 'doi:'10.1159/000499361.

16. Moresco L, Romantsik O, Calevo MG, Bruschettini M. Non-invasive respiratory support for the management of transient tachypnea of the newborn. Cochrane Database Syst Rev. 2020;4:D13231. 'doi:'10.1002/14651858.CD013231.pub2.

17. Nissen MD. Congenital and neonatal pneumonia. PAEDIATR RESPIR REV. 2007;8(3):195-203. 'doi:'10.1016/j.prrv.2007.07.001.

18. Chen L, Zhang Z. Bedside ultrasonography for diagnosis of pneumothorax. Quant Imaging Med Surg. 2015;5(4):618 - 23. 'doi:'10.3978/j.issn.2223-4292.2015.05.04.

19. Cattarossi L, Copetti R, Brusa G, Pintaldi S. Lung Ultrasound Diagnostic Accuracy in Neonatal Pneumothorax. CAN RESPIR J. 2016;2016:6515069. 'doi:'10.1155/2016/6515069.

20. Blank DA, Kamlin C, Rogerson SR, Fox LM, Lorenz L, Kane SC, Polglase GR, Hooper SB, Davis PG. Lung ultrasound immediately after birth to describe normal neonatal transition: an observational study, vol 22018.

21. Liu J, Copetti R, Sorantin E, Lovrenski J, Rodriguez-Fanjul J, Kurepa D, Feng X, Cattaross L, Zhang H, Hwang M, Yeh TF, Lipener Y, Lodha A, Wang JQ, Cao HY, Hu CB, Lyu GR, Qiu XR, Jia LQ, Wang XM, Ren XL, Guo JY, Gao YQ, Li JJ, Liu Y, Fu W, Wang Y, Lu ZL, Wang HW, Shang LL. Protocol and Guidelines for Point-of-Care Lung Ultrasound in Diagnosing Neonatal Pulmonary Diseases Based on International Expert Consensus. J Vis Exp. 2019(145). 'doi:'10.3791/58990.

22. de Souza TH, Nadal J, Peixoto AO, Pereira RM, Giatti MP, Soub A, Brandao MB. Lung ultrasound in children with pneumonia: interoperator agreement on specific thoracic regions. EUR J PEDIATR. 2019;178(9):1369-77. 'doi:'10.1007/s00431-019-03428-2.

23. Zong HF, Guo G, Liu J, Bao LL, Yang CZ. Using lung ultrasound to quantitatively evaluate pulmonary water content. Pediatr Pulmonol. 2020;55(3):729 - 39. 'doi:'10.1002/ppul.24635.

24. Cagini L, Andolfi M, Becattini C, Ranalli MG, Bartolucci F, Mancuso A, Vannucci J, Agnelli G, Puma F. Bedside sonography assessment of extravascular lung water increase after major pulmonary resection in non-small cell lung cancer patients. J THORAC DIS. 2018;10(7):4077-84. 'doi:'10.21037/jtd.2018.06.130.

25. Jambrik Z, Gargani L, Adamicza A, Kaszaki J, Varga A, Forster T, Boros M, Picano E. B-lines quantify the lung water content: a lung ultrasound versus lung gravimetry study in acute lung injury. ULTRASOUND MED BIOL. 2010;36(12):2004-10. 'doi:'10.1016/j.ultrasmedbio.2010.09.003.

26. Hooper SB, Te PA, Kitchen MJ. Respiratory transition in the newborn: a three-phase process. Arch Dis Child Fetal Neonatal Ed. 2016;101(3):F266-71. 'doi:'10.1136/archdischild-2013-305704.

27. Raimondi F, Migliaro F, Sodano A, Umbaldo A, Romano A, Vallone G, Capasso L. Can neonatal lung ultrasound monitor fluid clearance and predict the need of respiratory support? CRIT CARE. 2012;16(6):R220. 'doi:'10.1186/cc11865. 


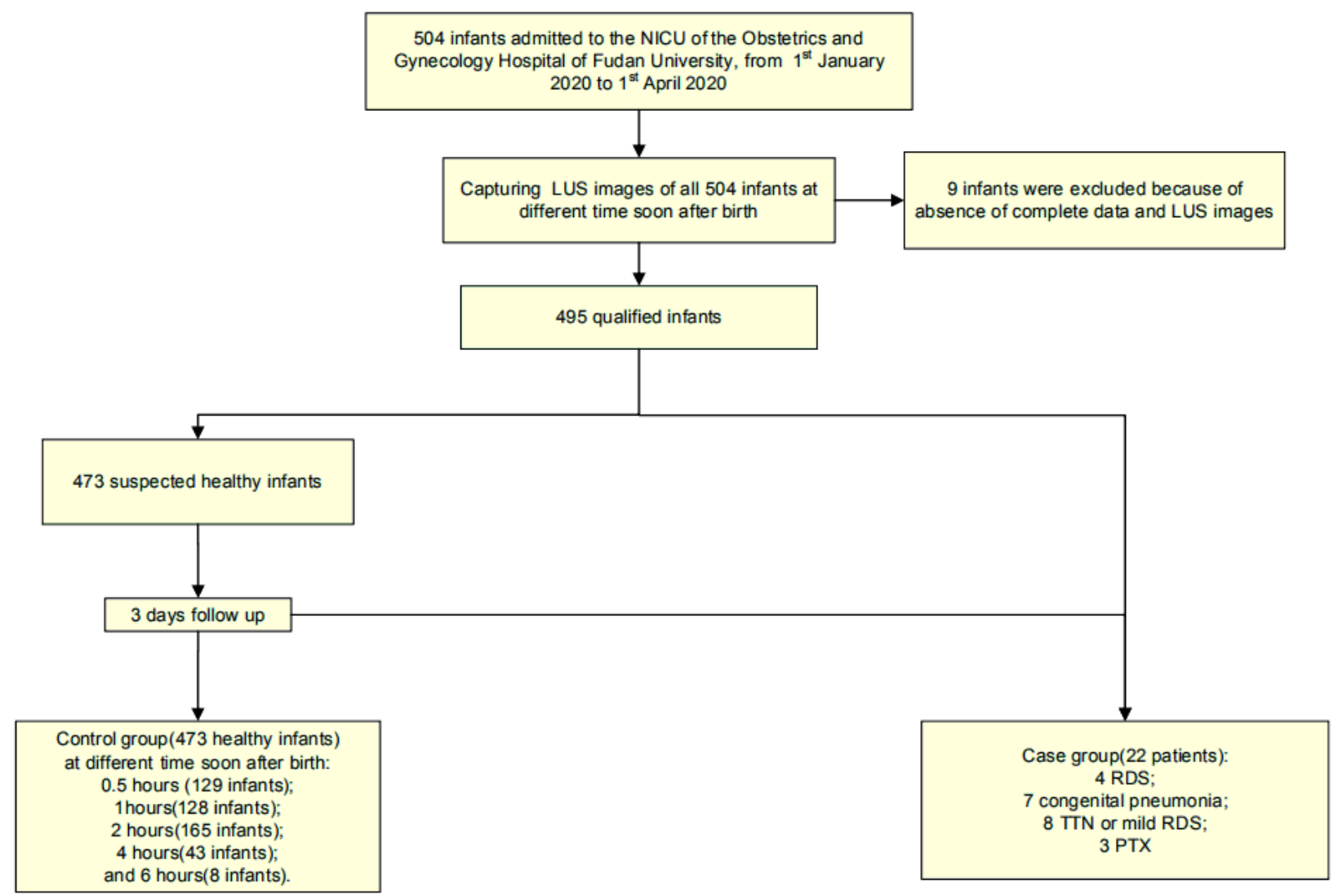

\section{Figure 1}

The participants flow chart. NICU: neonatal intensive care unit; LUS: lung ultrasound; RDS: respiratory distress; TTN: transient tachypnea of newborn PTX: pneumothorax 


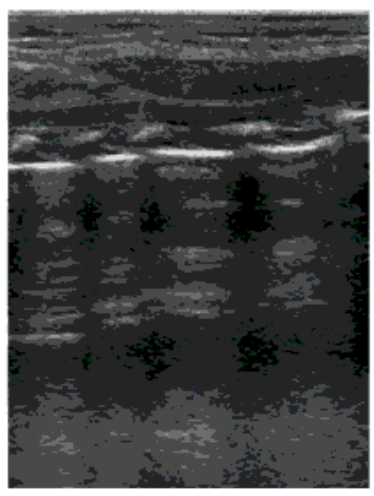

A

Pure A-line

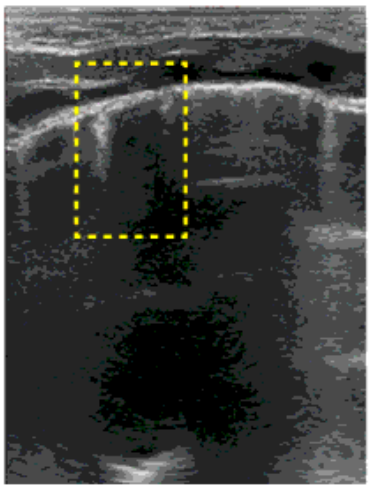

B

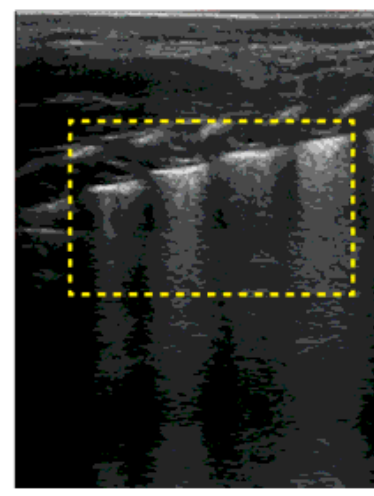

C

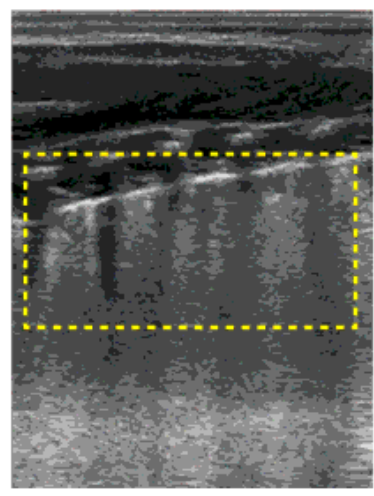

D

small amounts of DB moderate amounts of DB large amounts of DB

\section{LOW-RISK}

\section{HIGH-RISK}

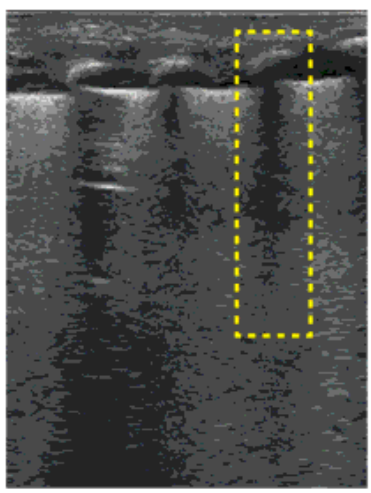

E

compact B-line

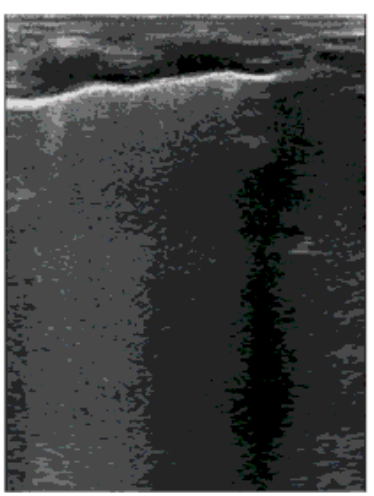

$\mathbf{F}$

Dense B-line

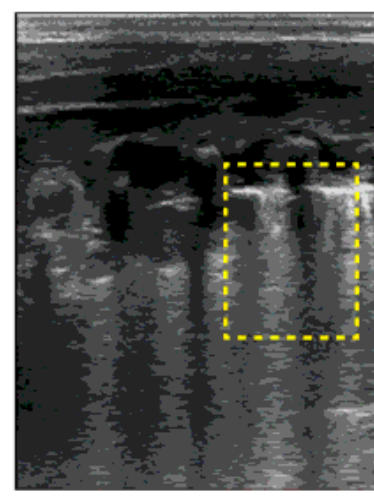

G

irregular consolidation with DB

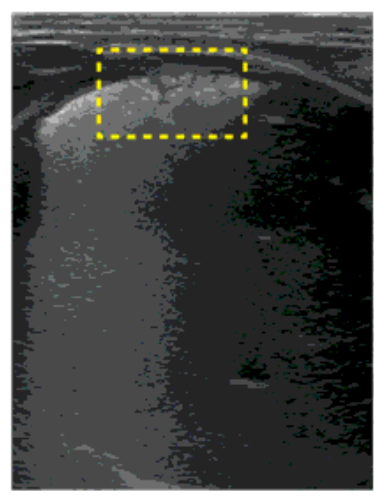

H

mild consolidation with air bronchograms

\section{Figure 2}

The 8 types of LUS images that may appear in healthy infants. DB: distributed B-lines, which means nearly every $B$ - lines can be discriminated against each other clearly. $(A)$ is mainly featured with pure Aline; $(B)(C)(D)$ are different kinds of B-line: (B) (small amount of B-line) is mainly characterized by distributed B-lines(yellow dotted box in A). (C) (moderate amount of DB)has more distributed B-lines but is not filled with them in the whole field. (D) (large amount of DB) presents a great number of distributed B-lines filling the field, but still can be discriminated against. (E) shows that the B-lines fused, but shadows of ribs can be seen(yellow dotted box in E). (F) is fused B-lines, and shadows of ribs can not be seen. $(G)$ represents consolidation companies with DB. $(H)$ is a small area of consolidation with air bronchograms. 


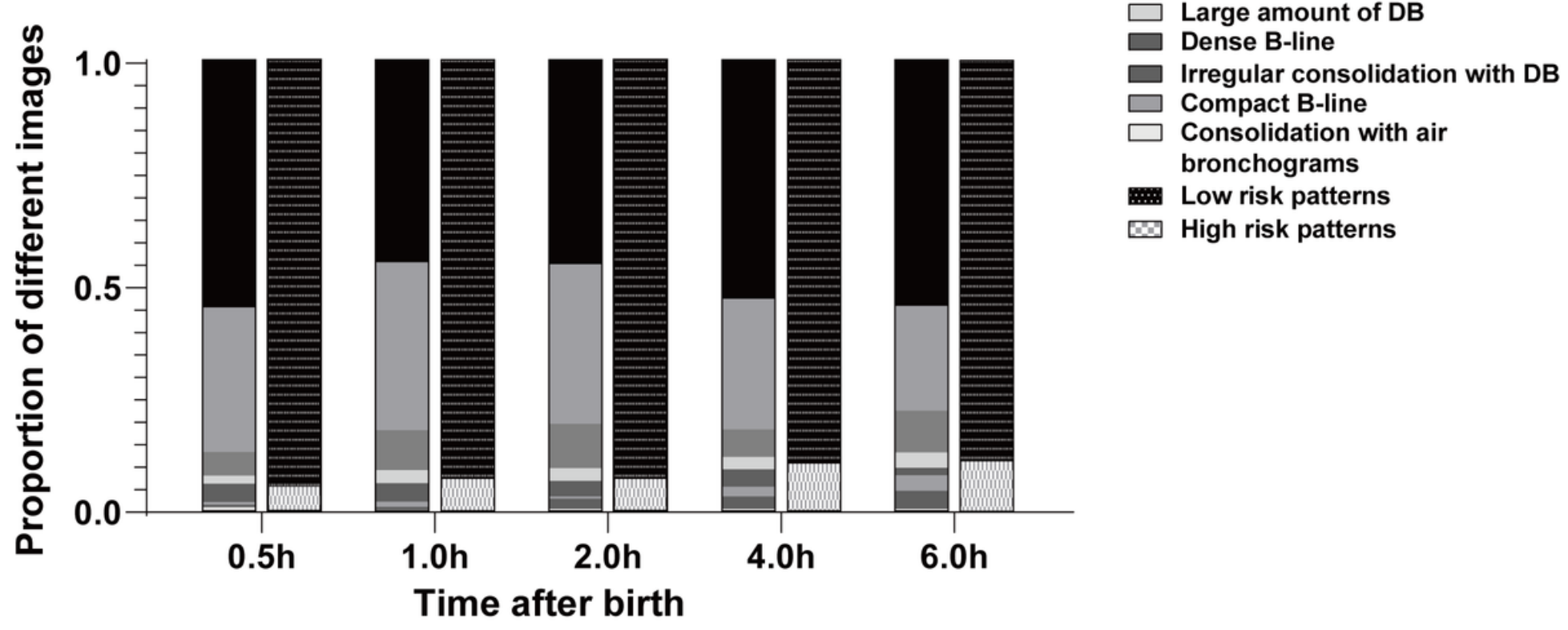

Figure 3

Variation of LUS images patterns shortly after birth. DB: distributed B-line. The ratio is calculated by dividing the numbers of each pattern by all 20 images for each infant. These images are captured according to the scanning protocol described in Fig. S1.

\section{Supplementary Files}

This is a list of supplementary files associated with this preprint. Click to download.

- OnlineFigS1.Png

- STROBEchecklistcasecontrol.pdf

- STARD2015Checklist.pdf 